\title{
Probiotics in viral infections, with a focus on COVID-19: A Systematic Review
}

\author{
Meraj Pourhossein ${ }^{1}$ and Amir Reza Moravejolahkami ${ }^{1}$ \\ ${ }^{1}$ Isfahan University of Medical Sciences
}

May 13, 2020

\begin{abstract}
COVID-19, a newly discovered virus disease, can be affected by probiotics. So, we tried to review the possible effects and associations of probiotics on viral infections focusing on SARS-CoV-2. We searched on PubMed, EMBASE, Google Scholar, Science Direct, Scopus and Web of Science up to May 2020 to identify interventional \& observational studies documenting the effects of probiotics strains on interleukins, virus titres, and antibody production with a focus on confirmed diagnosis of SARS-CoV-2 infection. From a total of 93 records, 21 studies were obtained and classified into three domains based on the efficacy of probiotics on 1)the level of interleukins $(n=7), 2)$ virus titres reduction $(n=2)$, and 3)antibody production ( $\mathrm{n}=12$ ). The suppuration of proinflammatory interleukins and type I interferon (IFN) production seemed to be the main anti-viral strategy of probiotics. Based on evidence, some probiotic strains may be useful in SARS-CoV-2 infection; randomized trials are needed to confirm these findings.
\end{abstract}

\section{Introduction:}

Severe Acute Respiratory Coronavirus 2 (SARS-CoV-2), a newly identified coronavirus with size of 60-140 $\mathrm{nm}$ similar to SARS-CoV (approximately $80 \%$ similar), caused the most severe pandemic disorder of $21_{\text {st }}$ century called COVID-19 (Lipsitch, Swerdlow, \& Finelli, 2020; Malik et al., 2020). SARS-CoV-2 attaches to Angiotensin Converting Enzyme 2 (ACE2) receptor with 10-20 folds higher affinity than SARS-CoV (X. Xu et al., 2020). Today, massive antibiotic therapy is considered for some COVID-19 patients that result in more susceptibility to subsequent infections (Mak, Chan, \& Ng, 2020); one of the most irritant complications is antibiotic-associated diarrhea (Wei et al., 2020). In this situation, probiotics may be a reasonable choice; they were defined as "Live microorganisms which when administered in adequate amounts confer a health benefit to the host" (Bahreini-Esfahani \& Moravejolahkami, 2020). on the other hand, Probiotics have shown useful effects on treatment and prevention of viral infections (Mousa, 2017) due to the proven immunomodulatory activity and ability to stimulate interferon production; gut-lung axis explains the possible relations between respiratory disorders and gut microbiome (Marsland, Trompette, \& Gollwitzer, 2015).

Recently, some COVID-19 patients showed microbial dysbiosis with decreased Lactobacillus and Bifidobacterium (K. Xu et al., 2020). Several different probiotics, including Lactobacillus acidophilus, Bifidobacterium and Saccharomyces boulardii, along with minerals \& vitamins were given to a COVID-19 case; this type of nutritional support lowered the complication of massive antibiotic therapy (Horowitz, Freeman, \& Bruzzese, 2020). As well, COVID-19-like symptoms disappeared after two days administration of oral probiotic in a 9 years-old boy (Ji et al., 2020). Results of a case series consist of 62 SARS-CoV2 infected patients in Zhejiang province were interesting; probiotics were administered as adjunct (X.-W. Xu et al., 2020). Other reports showed significant effect of probiotics; Compared to patients with non-severe disease, patients with severe disease had significantly more frequency to receive probiotics tablets $(87.5 \%$ vs $40.4 \%, \mathrm{p}=0.037)$ (Xiufeng Jiang et al., 2020). Results from another research suggested concurrent use of probiotics in COVID-19 patients to decrease the risk for Candida albicans (caused by prophylaxis with azithromycin) (Block, 2020). 
Up to April 2020, two clinical trials have been registered regarding the probiotics supplementation in COVID19 patients (Lythgoe \& Middleton, 2020), however, more information is needed for designing research protocols. We tried to summarize the possible relationships between probiotics and viral infections especially SARS-CoV-2-related literature in this systematic review.

\section{Methods:}

\subsection{Protocol registration}

The protocol of this systematic review has been registered on PROSPERO website (www.crd.york.ac.uk/PROSPERO) (Registration ID. CRD42020181453) developed based on the Preferred Reporting Items for Systematic Reviews and Meta-Analyses (PRISMA) statement guidelines (Liberati et al., 2009).

\subsection{Search strategy}

A comprehensive search of the literature was conducted in the following databases up to May 1, 2020: PubMed, EMBASE, Google Scholar, Science Direct, Scopus, and Web of Science. All citations were imported into a bibliographic database (EndNote X8.1; Thomson Reuters) and duplicates were removed. This search process was handled independently by one author (AR.M).

The used search string - based on suitable MESH and non-MESH keywords - were: ("COVID-19" OR "SARS-CoV-2" OR "Severe Acute Respiratory Coronavirus 2" OR "coronavirus" OR "virus disease" OR "viral infection" OR "virus") AND ("virus titres" OR "titres" OR "interleukin" OR "IL-6" OR "IL-17" OR "antibody" OR "IgG" OR "IgA" OR "interferon" OR "INF") AND ("Synbiotics" OR "Probiotics" OR "Prebiotics")). Moreover, bibliographies of all published reviews and studies were assessed for additional relevant papers.

\subsection{Study selection criteria, data extraction and quality assessment}

Title, abstract and then full-text of all articles were screened to select \& extract studies that investigated the effect of probiotics on viral infections (with emphasis on SARS-CoV-2) in English language (all research types with both human and animal origin). We excluded HIV papers, due to not being directly related to respiratory viral infections. Data extraction from primary articles was performed by one author (AR.M) by using a standardized form. A second author checked the accuracy of the data extracted (M.P). Data collected from the studies included authors' family name, type of study, probiotic strains and dose, sample size, and overall outcomes. The Cochrane Collaboration criteria for assessing risk of bias were applied to assess the quality of the studies included in the review (Higgins \& Green, 2011).

\section{Results}

A diagram showed the details of included studies in Figure 1. A total of 93 recorded were identified initially from selected databases. After excluding duplicates and articles that did not meet the inclusion criteria, we obtained 48 articles with full-texts which were read for further evaluation, where another 27 were excluded due to insufficient data and duplication. Overall, we included 21 articles for qualitative analysis.

\subsection{Major effects of probiotics in viral infections:}

COVID-19-related probiotic strains - up to May 2020 - were summarized in Figure 2 . As well, the administered probiotics in other viral infections were briefly displayed in Figure 3 .

\subsubsection{Effects on Level of Interleukins}

Oral Lactobacillus casei ATCC 39392 vaccine polarized Th2 cell immunity and stimulated IL-17 expression against transmissible gastroenteritis coronavirus (TGEV) (Xinpeng Jiang et al., 2016), however, an anti interleukin (IL)-17 effect was observed forBifidobacterium animalis (Hüseyin Sancar Bozkurt \& Quigley, 2019). In another study, the probiotic strain "heat-killed Lactobacillus plantarum L-137" was introduced as an IL-12 inducer in mice (Murosaki et al., 1998). 
In a trial conducted by kimmel et al, 10 healthy Subjects consumed 1 capsule/day of Bacillus coagulans GBI30, 6086 (Ganeden $B C^{30}$ ) for 28 days and returned for post-treatment immunological markers evaluation; IL-6, IL-8, and TNF-alpha levels were increased after exposure to a strain of adenovirus (AdenoVI) and influenza A (H3N2 Texas strain; FluTex). IL-1beta levels also increased after exposure to AdenoVI but were reduced after ex vivo exposure to FluTex (Kimmel, Keller, Farmer, \& Warrino, 2010).

Some hypotheses indicated that oral administration of theStreptococcus salivarius K12 strain, through a still not perfectly clear molecular mechanism, reduced IL-8 plasma concentrations and increased salivary interferon- $\gamma$. These modulations may also realistically account for anti-inflammatory and antiviral activity, which would then be added to the antibacterial action of the K12 strain (Wescombe, Hale, Heng, \& Tagg, 2012).

Coronaviruses may also vulnerable to probiotics. Chi et al. after infecting swine testicle (ST) cells with TGEV administeredEnterococcus faecium NCIMB 10415 ; as a result, production of nitric oxide increased and expression of IL-6 \& 8 in the cells treated with E. faecium was elevated (Chai et al., 2013). Another research claimed that Enterococcus faecium HDRsEf1 could significantly downregulate the mRNA level of pro-inflammatory factors IL-13, IL-6, IL-8, IL-12, IL-17, and TNF-a (Tian et al., 2016).

\subsubsection{Effects on Virus Titres Reduction}

21 days feeding yogurt fermented with Lactobacillus delbrueckii ssp. bulgaricus OLL1073R-1 (1073R-1) significantly decreased influenza virus titres in infected mice (Nagai, Makino, Ikegami, Itoh, \& Yamada, 2011).

Results of an animal study in mice conducted by Maeda et al. showed thatheat-killed Lactobacillus plantarum L-137 can lower the viral titres of influenza virus A/FM/1/47 (H1N1, a mouse-adapted strain) (Maeda et al., 2009). Other researchers demonstrated this effect on humans (Arimori et al., 2012). Contrary, Another probiotic "heat-treated Enterococcus faecalis strain FK-23" during 6-36 months administration for 39 adult HCV-positive subjects could reduce alanine aminotransferase (ALT) and aspartate transaminase (AST) with no significant effect on viral load (Oo et al., 2016).

\subsubsection{Effects on Antibody Production}

Consuming 1 capsule/day of Bacillus coagulans GBI-30, 6086 (GanedenBC ${ }^{30}$ ) for 28 days increased CD3+CD69+ cells and interferon-gamma (IFN-gamma) levels (Kimmel et al., 2010). Moreover,Lactobacillus delbrueckii ssp. bulgaricus OLL1073R-1 (1073R-1)increased anti-influenza virus (A/PR/8/34 (H1N1)) antibodies (IgA, IgG1) in animal model (Nagai et al., 2011). Interestingly, heat-killed Lactobacillus plantarum $L-137$ can act as an IFN- $\beta$ inducer (Maeda et al., 2009).

Lactobacillus casei shirota, another potential probiotic, was capable to reduce plasma antibody titres in cytomegalovirus and Epstein-Barr virus infected people through modulation the activity of natural killer (NK) cells (Kawai \& Akira, 2006); but it had not any therapeutic effect on norovirus-induced gastroenteritis (Nagata et al., 2011).

Oral administration of yogurt containing Lactobacillus gasseri SBT2055 (LG2055) in healthy adult subjects potentiated vaccine-specific antibody production against A/H1N1 and B influenza viruses (Nishihira et al., 2018). In another trial, Titres against the influenza B strain increased significantly in group receiving Lactobacillus casei DN-114 001 (CNCMI-1518) for 13 weeks (Boge et al., 2009).

Some other strains such as Lactobacillus rhamnosus ( $L G G$ ), when administered for 4 weeks to children with gastroenteritis who were positive for either rotavirus or Cryptosporidium species in stool, caused a significant increase in serum immunoglobulin (Ig) G levels occurred (Sindhu et al., 2014). According to evidence, the titres of vaccine-specific IgG, IgG1, and IgG3 in plasma as well as that of vaccine-specific secretory $\operatorname{IgA}$ in saliva were significantly greater in those who received both probiotics "Bifidobacterium animalis ssp. lactis and Lactobacillus paracasei ssp. Paracasei $\left(10^{9}\right.$ CFU for 6 weeks)" (Rizzardini et al., 2012). Furthermore, 172 full-term infants that were fed by probiotics formula $\left(10^{6} \mathrm{CFU} / \mathrm{g}\right.$ Bifidobacterium animalis ssp. lactis; 
Bb12) had more anti-poliovirus-specific IgA in the feces sample, but not anti-rotavirus-specific IgA (Holscher et al., 2012).

Akatsu et al. (Akatsu et al., 2013) highlighted a significant association between Bifidobacterium and increased immune function and intestinal microbiota in the elderly; administration ofBifidobacterium longum( BB536) for 5 weeks resulted in an increase of serum IgA. BB536 intake did not significantly affect hemagglutination inhibition titres in response to influenza vaccine. NK cell activity did not also decrease significantly in probiotics group.

Interestingly, Receiving a jelly containing 10 billionheat-killed Lactobacillus paracasei MCC1849 cells for 6 weeks could improve the antibody responses to the A/H1N1 and B antigens in [?] 85 years of age subgroup $(\mathrm{n}=27)$, anyway, the overall results were not significant compared with placebo (Maruyama et al., 2016).

\section{Discussion:}

Here, we reviewed the literature regarding several probiotics based on the papers which described single probiotics in clinical trial to avoid the crosstalk or mutual interference between probiotics. To the best of our knowledge, this is the first systematic review reporting probiotics-related findings in COVID-19 pandemic. Probiotic strains likeLactobacillus plantarum, Lactobacillus casei,Bifidobacterium animalis, Bacillus coagulans, Streptococcus salivarius, and Enterococcus faecium had inhibitory effects on proinflammatory interleukins. Moreover, several conventional probiotics (Lactobacillus gasseri, Lactobacillus rhamnosus, Bifidobacterium longum ) potentially increased the level of antibodies.

Coronaviruses (CoVs) involve a large family of surrounded, positive-stranded RNA viruses that infect a wide range of animal hosts as well as humans. The most common representatives are porcine respiratory $\mathrm{CoV}$, porcine transmissible gastroenteritis virus, porcine epidemic diarrhoea virus, canine $\mathrm{CoV}$, human $\mathrm{CoVs}$, severe acute respiratory syndrome-associated $\mathrm{CoV}$, murine hepatitis virus (MHV), avian $\mathrm{CoV}$ infectious bronchitis virus (IBV) and turkey $\mathrm{CoV}$ (TCoV). The most recognized coronavirus, the Middle East respiratory syndrome virus, was found in Africa and Asia (Siddell et al., 1983). At the present, antivirals have been developed for management of the $\mathrm{CoV}$-induced infections, however, the treatment process is affected by other determinants. For example, some data are available on the association of COVID-19 and intestinal microbiota. Lactobacilli andBifidobacteria are two conventional probiotics that can really tip the balance of a diverse gut ecosystem in combating COVID-19 (K. Xu et al., 2020).

There are some mechanisms explaining the antiviral impacts of probiotics. A brief report (Hüseyin S Bozkurt, 2020) claimed that high-single dose endoscopic administration of appropriate and reliableBifidobacterium Infantis (BB-12), as potential probiotic, or use of these strains' lipopolysaccharide membranes in vaccine development especially in patients with gastrointestinal symptoms (diarrhea, abdomen pain, vomiting) can result in more therapeutic and preventive effects in Coronavirus-infected patients (K. Bozkurt et al., 2019; Schiavi et al., 2016). Consequently,Bifidobacterium animalis theoretically can inhibit the replication of coronaviruses by reducing ER stress-related autophagy, especially the inositol-requiring enzyme 1 (IRE1) pathway, over its anti interleukin-17 effect (Hüseyin Sancar Bozkurt \& Quigley, 2019). Apparently, the main pathogenic mechanism that makes viral infection-induced pneumonia seems to be a "cytokine storm"; IL-6 is the main proinflammatory marker in this regard (Angeletti et al., 2020). Anyway, the state of knowledge regarding the immunomodulatory effects of probiotics has recently advanced.

Some probiotics have potency to induce the interferon (IFN) production pathways. Lactococcus lactis JCM5805 can activate human Plasmacytoid dendritic cells (pDCs) in vitro; pDCs play a crucial role in antiviral immunity as proficient type I IFN producing cells (IPCs) (Siegal et al., 1999; Trinchieri \& Santoli, 1978) and mucosal T cell independent IgA production (Tezuka et al., 2011). pDC-derived type I IFNs can indirectly inhibit viral replication and spread (Theofilopoulos, Baccala, Beutler, \& Kono, 2005), and activation of NK cells (Tezuka et al., 2011). Two distinct types of Toll-like receptors (TLR7 and TLR9) expressed by pDCs - have some molecular role (Gilliet, Cao, \& Liu, 2008); TLR7 recognizes the microbial RNA of virus (Lee, Lund, Ramanathan, Mizushima, \& Iwasaki, 2007; Lund et al., 2004), and TLR9 senses the ssDNA containing CpG motifs (Bauer et al., 2001; Latz et al., 2007). Upon sensing the viral nucleic acid, 
interferon regulatory factor 7 (IRF7) is activated, phosphorylated, and translocated into the nucleus to begin the transcription of type I IFNs (Kawai et al., 2004). In conclusion, this strain had many important roles in both innate immunity and adaptive immunity; as well, several human-based trials confirmed this observation (influenza virus as main target) (Fujii et al., 2017; Jounai, Sugimura, Ohshio, \& Fujiwara, 2015; Shibata et al., 2016). Furthermore, some probiotic strains such as Enterococcus faecium HDRsEf1 could significantly downregulate the mRNA level of TLR4, TLR5, TLR7, and TLR8 (Tian et al., 2016).

As discussed earlier, there have also been negative reports against probiotics. According to scRNA-Seq analysis, Feng et al. found that the SARS-Cov-2 receptor, ACE2, could be elevated in the presence of both invasive bacteria Salmonella Enterica and its counterpart,Segmented Filamentous Bacteria as probiotics in the mouse small intestine (Liang et al., 2020) and human enterocytes (Feng, Wang, \& Qi, 2020). in another study Lactobacillus acidophilus andBacillus clausii also failed to decrease the coronavirus receptors expression in the murine small intestine compared to control and post Salmonella infection (Pradhan et al., 2019).

In total, the US Food and Drug Administration classifies these as GRAS (Generally Recognized As Safe) organisms, so the risks of probiotics administration seem to be low, although the amount and type of strains are also considerable (Askari \& Moravejolahkami, 2019; Moravejolahkami \& Chitsaz, 2019). Notably, unconfirmed prescription of conventional probiotics for COVID-19 is not recommended until further investigations regarding the pathogenesis of SARS-CoV-2 and its effect on gut microbiota were published.

A few limitations of this review shall be highlighted; systematic review table was not designed due to inconsistent data of relevant studies; COVID-19 papers did not report the required detail. Moreover, metaanalysis has not been performed due to heterogeneity of studies, especially in relation to published data for SARS-CoV-2. Secondly, we excluded a large number of research on supplementation of different nutrients, which were related to immune function. However, the main strength of the current study was that we performed an exclusive investigation for diseases of the same origin i.e. virus disease, especially COVID-19 for first time.

\section{Conclusion}

We described the efficacy of probiotics for the prevention or treatment of viral-based infectious diseases; more than 20 strains improved the anti-inflammatory interleukins and anti-body production against viruses. Moreover, virus titres were lowered after probiotics supplementation periods. The large number of viral species and their subtypes as well as the high mutation rate of viruses do not allow scientists to discover appropriate vaccines and antiviral drugs, so, the administration of pro/prebiotics - as immune function modulators and antibiotic-related side effects removers - is recommended. Although further detailed research is necessary, the authors recommend researchers/physicians/dietitians to use probiotics as more rational adjunctive option in COVID-19 pandemic, especially in mechanically ventilated patients.

\section{Acknowledgement}

We thank all the nurses, doctors, clinicians and researchers during COVID-19 pandemic.

\section{Disclaimer statements}

\section{Contributors}

M. Pourhossein: Supervision, Critical Reviewing and Editing the Final Version of the Manuscript.AR. Moravejolahkami: Conceptualization, Writing-Original draft preparation and Software, Assessing Study Eligibility and Conducting Quality Assessments, Validation, Visualization, Investigation.

\section{Funding}

The authors declare no support from any commercial organization for the submitted study.

\section{Conflicts of interest}


None of the authors had a conflict of interest.

\section{Figure Legends:}

Figure 1. Flow diagram of the different phases of systematic review.

Figure 2. The probiotics strains that have been reported in the context of COVID-19 (up to May 7, 2020).

Figure 3. Evidence-based efficacy of some probiotic strains in viral infections.

\section{References:}

Akatsu, H., Iwabuchi, N., Xiao, J. z., Matsuyama, Z., Kurihara, R., Okuda, K., . . . Maruyama, M. (2013). Clinical effects of probiotic Bifidobacterium longum BB536 on immune function and intestinal microbiota in elderly patients receiving enteral tube feeding.Journal of Parenteral and Enteral Nutrition, 37 (5), 631-640.

Angeletti, S., Benvenuto, D., Bianchi, M., Giovanetti, M., Pascarella, S., \& Ciccozzi, M. (2020). COVID2019: the role of the nsp2 and nsp3 in its pathogenesis. Journal of medical virology .

Arimori, Y., Nakamura, R., Hirose, Y., Murosaki, S., Yamamoto, Y., Shidara, O., . . . Yoshikai, Y. (2012). Daily intake of heat-killed Lactobacillus plantarum L-137 enhances type I interferon production in healthy humans and pigs. Immunopharmacology and immunotoxicology, 34 (6), 937-943.

Askari, G., \& Moravejolahkami, A. R. (2019). Synbiotic Supplementation May Relieve Anterior Uveitis, an Ocular Manifestation in Behcet's Syndrome. The American journal of case reports, 20 , 548.

Bahreini-Esfahani, N., \& Moravejolahkami, A. R. (2020). Can Synbiotic Dietary Pattern Predict Lactobacillales Strains in Breast Milk?Breastfeeding Medicine .

Bauer, S., Kirschning, C. J., Hacker, H., Redecke, V., Hausmann, S., Akira, S., . . . Lipford, G. B. (2001). Human TLR9 confers responsiveness to bacterial DNA via species-specific CpG motif recognition. Proceedings of the national academy of sciences, 98 (16), 9237-9242.

Block, J. (2020). High Risk COVID-19: Potential Intervention at Multiple Points in the COVID-19 Disease Process Via Prophylactic Treatment with Azithromycin or Bee Derived Products.

Boge, T., Remigy, M., Vaudaine, S., Tanguy, J., Bourdet-Sicard, R., \& Van Der Werf, S. (2009). A probiotic fermented dairy drink improves antibody response to influenza vaccination in the elderly in two randomised controlled trials. Vaccine, 27 (41), 5677-5684.

Bozkurt, H. S. (2020). PROBIOTIC BACTERIA AGAINST THE COVID-19. doi:10.31219/osf.io/hz26y

Bozkurt, H. S., \& Quigley, E. M. (2019). Bifidobacteria and Mucosal-Associated Invariant T (MAIT) Cells: A New Approach to Colorectal Cancer Prevention? Gastrointestinal Disorders, 1 (2), 266-272.

Bozkurt, K., Denktaş, C., Özdemir, O., Altındal, A., Avdan, Z., \& Bozkurt, H. (2019). Charge Transport in Bifidobacterium animalis subsp. lactis BB-12 under the various Atmosphere. arXiv preprint arXiv:1901.10765 .

Chai, W., Burwinkel, M., Wang, Z., Palissa, C., Esch, B., Twardziok, S., . . . Schmidt, M. F. (2013). Antiviral effects of a probiotic Enterococcus faecium strain against transmissible gastroenteritis coronavirus. Archives of virology, 158 (4), 799-807.

Feng, Z., Wang, Y., \& Qi, W. (2020). The small intestine, an underestimated site of SARS-CoV-2 infection: from red queen effect to probiotics. Preprints .

Fujii, T., Jounai, K., Horie, A., Takahashi, H., Suzuki, H., Ohshio, K., . . . Yamamoto, N. (2017). Effects of heat-killed Lactococcus lactis subsp. lactis JCM 5805 on mucosal and systemic immune parameters, and antiviral reactions to influenza virus in healthy adults; a randomized controlled double-blind study. Journal of Functional Foods, 35, 513-521. 
Gilliet, M., Cao, W., \& Liu, Y.-J. (2008). Plasmacytoid dendritic cells: sensing nucleic acids in viral infection and autoimmune diseases. Nature Reviews Immunology, 8 (8), 594-606.

Higgins, J. P., \& Green, S. (2011). Cochrane handbook for systematic reviews of interventions (Vol. 4): John Wiley \& Sons.

Holscher, H. D., Czerkies, L. A., Cekola, P., Litov, R., Benbow, M., Santema, S., . . . Saavedra, J. M. (2012). Bifidobacterium lactis Bb12 enhances intestinal antibody response in formula-fed infants: a randomized, double-blind, controlled trial. Journal of parenteral and enteral nutrition, 36 , 106S-117S.

Horowitz, R. I., Freeman, P. R., \& Bruzzese, J. (2020). Efficacy of glutathione therapy in relieving dyspnea associated with COVID-19 pneumonia: A report of 2 cases. Respiratory Medicine Case Reports , 101063.

Ji, L.-N., Chao, S., Wang, Y.-J., Li, X.-J., Mu, X.-D., Lin, M.-G., \& Jiang, R.-M. (2020). Clinical features of pediatric patients with COVID-19: a report of two family cluster cases. World Journal of Pediatrics , $1-4$.

Jiang, X., Hou, X., Tang, L., Jiang, Y., Ma, G., \& Li, Y. (2016). A phase trial of the oral Lactobacillus casei vaccine polarizes Th2 cell immunity against transmissible gastroenteritis coronavirus infection. Applied microbiology and biotechnology, 100 (17), 7457-7469.

Jiang, X., Tao, J., Wu, H., Wang, Y., Zhao, W., Zhou, M., . . . Zhu, F. (2020). Clinical features and management of severe COVID-19: A retrospective study in Wuxi, Jiangsu Province, China. medRxiv .

Jounai, K., Sugimura, T., Ohshio, K., \& Fujiwara, D. (2015). Oral administration of Lactococcus lactis subsp. lactis JCM5805 enhances lung immune response resulting in protection from murine parainfluenza virus infection. PloS one, 10 (3).

Kawai, T., \& Akira, S. (2006). Innate immune recognition of viral infection. Nature immunology, 7 (2), 131-137.

Kawai, T., Sato, S., Ishii, K. J., Coban, C., Hemmi, H., Yamamoto, M., . . . Uematsu, S. (2004). Interferon$\alpha$ induction through Toll-like receptors involves a direct interaction of IRF7 with MyD88 and TRAF6. Nature immunology, 5 (10), 1061-1068.

Kimmel, M., Keller, D., Farmer, S., \& Warrino, D. (2010). A controlled clinical trial to evaluate the effect of GanedenBC (30) on immunological markers. Methods and findings in experimental and clinical pharmacology, 32 (2), 129-132.

Latz, E., Verma, A., Visintin, A., Gong, M., Sirois, C. M., Klein, D. C., . . . Duprex, W. P. (2007). Ligand-induced conformational changes allosterically activate Toll-like receptor 9. Nature immunology, 8 (7), 772-779.

Lee, H. K., Lund, J. M., Ramanathan, B., Mizushima, N., \& Iwasaki, A. (2007). Autophagy-dependent viral recognition by plasmacytoid dendritic cells. Science, 315 (5817), 1398-1401.

Liang, W., Feng, Z., Rao, S., Xiao, C., Xue, X., Lin, Z., . . . Qi, W. (2020). Diarrhoea may be underestimated: a missing link in 2019 novel coronavirus. Gut .

Liberati, A., Altman, D. G., Tetzlaff, J., Mulrow, C., Gøtzsche, P. C., Ioannidis, J. P., . . Moher, D. (2009). The PRISMA statement for reporting systematic reviews and meta-analyses of studies that evaluate health care interventions: explanation and elaboration. Annals of internal medicine, 151 (4), W-65-W-94.

Lipsitch, M., Swerdlow, D. L., \& Finelli, L. (2020). Defining the epidemiology of Covid-19 - studies needed. New England Journal of Medicine, 382 (13), 1194-1196.

Lund, J. M., Alexopoulou, L., Sato, A., Karow, M., Adams, N. C., Gale, N. W., . . Flavell, R. A. (2004). Recognition of single-stranded RNA viruses by Toll-like receptor 7. Proceedings of the National Academy of Sciences, 101 (15), 5598-5603. 
Lythgoe, M. P., \& Middleton, P. (2020). Ongoing clinical trials for the management of the COVID-19 pandemic. Trends in Pharmacological Sciences .

Maeda, N., Nakamura, R., Hirose, Y., Murosaki, S., Yamamoto, Y., Kase, T., \& Yoshikai, Y. (2009). Oral administration of heat-killed Lactobacillus plantarum L-137 enhances protection against influenza virus infection by stimulation of type I interferon production in mice.International immunopharmacology, 9 (9), $1122-1125$.

Mak, J. W., Chan, F. K., \& Ng, S. C. (2020). Probiotics and COVID-19: one size does not fit all. The Lancet Gastroenterology \& Hepatology .

Malik, Y. S., Sircar, S., Bhat, S., Vinodhkumar, O. R., Tiwari, R., Sah, R., . . Dhama, K. (2020). Emerging Coronavirus Disease (COVID-19), a pandemic public health emergency with animal linkages: Current status update.

Marsland, B. J., Trompette, A., \& Gollwitzer, E. S. (2015). The gut-lung axis in respiratory disease. Annals of the American Thoracic Society, 12 (Supplement 2), S150-S156.

Maruyama, M., Abe, R., Shimono, T., Iwabuchi, N., Abe, F., \& Xiao, J.-Z. (2016). The effects of nonviable Lactobacillus on immune function in the elderly: a randomised, double-blind, placebo-controlled study.International journal of food sciences and nutrition, 67 (1), 67-73.

Moravejolahkami, A., \& Chitsaz, A. (2019). Mediterranean-Style Diet Co-Supplemented with Synbiotics Improved Quality of Life, Fatigue and Disease Activity in Five Secondary Progressive Multiple Sclerosis Patients. Ann Med \& Surg Case Rep: AMSCR, 2019 (02).

Mousa, H. A.-L. (2017). Prevention and treatment of influenza, influenza-like illness, and common cold by herbal, complementary, and natural therapies. Journal of evidence-based complementary $\&$ alternative medicine, 22 (1), 166-174.

Murosaki, S., Yamamoto, Y., Ito, K., Inokuchi, T., Kusaka, H., Ikeda, H., \& Yoshikai, Y. (1998). Heat-killed Lactobacillus plantarum L-137 suppresses naturally fed antigen-specific IgE production by stimulation of IL-12 production in mice. Journal of allergy and clinical immunology, 102 (1), 57-64.

Nagai, T., Makino, S., Ikegami, S., Itoh, H., \& Yamada, H. (2011). Effects of oral administration of yogurt fermented with Lactobacillus delbrueckii ssp. bulgaricus OLL1073R-1 and its exopolysaccharides against influenza virus infection in mice. International immunopharmacology, 11 (12), 2246-2250.

Nagata, S., Asahara, T., Ohta, T., Yamada, T., Kondo, S., Bian, L., . . . Nomoto, K. (2011). Effect of the continuous intake of probiotic-fermented milk containing Lactobacillus casei strain Shirota on fever in a mass outbreak of norovirus gastroenteritis and the faecal microflora in a health service facility for the aged. British Journal of Nutrition, 106 (4), 549-556.

Nishihira, J., Nishimura, M., Moriya, T., Sakai, F., Kabuki, T., \& Kawasaki, Y. (2018). Lactobacillus Gasseri Potentiates Immune Response Against Influenza Virus Infection Immunity and Inflammation in Health and Disease (pp. 249-255): Elsevier.

Oo, K. M., AYELWIN, A., Kyaw, Y. Y., Tun, W. M., Fukada, K., Goshima, A., . . Okada, S. (2016). Safety and long-term effect of the probiotic FK-23 in patients with hepatitis C virus infection. Bioscience of microbiota, food and health , 2015-2024.

Pradhan, B., Guha, D., Naik, A. K., Banerjee, A., Tambat, S., Chawla, S., . . . Aich, P. (2019). Probiotics L. acidophilus and B. clausii modulate gut microbiota in Th1-and Th2-biased mice to ameliorate salmonella typhimurium-induced diarrhea. Probiotics and antimicrobial proteins, 11 (3), 887-904.

Rizzardini, G., Eskesen, D., Calder, P. C., Capetti, A., Jespersen, L., \& Clerici, M. (2012). Evaluation of the immune benefits of two probiotic strains Bifidobacterium animalis ssp. lactis, BB-12@ and Lactobacillus 
paracasei ssp. paracasei, L. casei 431@ in an influenza vaccination model: a randomised, double-blind, placebo-controlled study.British journal of nutrition, 107 (6), 876-884.

Schiavi, E., Gleinser, M., Molloy, E., Groeger, D., Frei, R., Ferstl, R., . . . Moriarty, T. F. (2016). The surface-associated exopolysaccharide of Bifidobacterium longum 35624 plays an essential role in dampening host proinflammatory responses and repressing local TH17 responses. Appl. Environ. Microbiol., 82 (24), 7185-7196.

Shibata, T., Kanayama, M., Haida, M., Fujimoto, S., Oroguchi, T., Sata, K., . . Kondo, M. (2016). Lactococcus lactis JCM5805 activates anti-viral immunity and reduces symptoms of common cold and influenza in healthy adults in a randomized controlled trial. Journal of Functional Foods, 24, 492-500.

Siddell, S., Anderson, R., Cavanagh, D., Fujiwara, K., Klenk, H., Macnaughton, M., . . Van der Zeijst, B. (1983). Coronaviridae.Intervirology, 20 (4), 181-189.

Siegal, F. P., Kadowaki, N., Shodell, M., Fitzgerald-Bocarsly, P. A., Shah, K., Ho, S., . . Liu, Y.-J. (1999). The nature of the principal type 1 interferon-producing cells in human blood. Science, 284 (5421), 1835-1837.

Sindhu, K. N., Sowmyanarayanan, T. V., Paul, A., Babji, S., Ajjampur, S. S., Priyadarshini, S., . . Ward, H. D. (2014). Immune response and intestinal permeability in children with acute gastroenteritis treated with Lactobacillus rhamnosus GG: a randomized, double-blind, placebo-controlled trial. Clinical infectious diseases, 58 (8), 1107-1115.

Tezuka, H., Abe, Y., Asano, J., Sato, T., Liu, J., Iwata, M., \& Ohteki, T. (2011). Prominent role for plasmacytoid dendritic cells in mucosal T cell-independent IgA induction. Immunity, 34 (2), 247-257.

Theofilopoulos, A. N., Baccala, R., Beutler, B., \& Kono, D. H. (2005). Type I interferons $(\alpha / \beta)$ in immunity and autoimmunity. Annu. Rev. Immunol., 23, 307-335.

Tian, Z., Yang, L., Li, P., Xiao, Y., Peng, J., Wang, X., . . . Shi, D. (2016). The inflammation regulation effects of Enterococcus faecium HDRsEf1 on human enterocyte-like HT-29 cells. Animal cells and systems, 20 (2), 70-76.

Trinchieri, G., \& Santoli, D. (1978). Anti-viral activity induced by culturing lymphocytes with tumor-derived or virus-transformed cells. Enhancement of human natural killer cell activity by interferon and antagonistic inhibition of susceptibility of target cells to lysis. The Journal of experimental medicine, 147 (5), 1314-1333.

Wei, X.-S., Wang, X., Niu, Y.-R., Ye, L.-L., Peng, W.-B., Wang, Z.-H., . . . Ma, W.-L. (2020). Diarrhea is associated with prolonged symptoms and viral carriage in COVID-19. Clinical Gastroenterology and Hepatology .

Wescombe, P. A., Hale, J. D., Heng, N. C., \& Tagg, J. R. (2012). Developing oral probiotics from Streptococcus salivarius. Future microbiology, 7 (12), 1355-1371.

Xu, K., Cai, H., Shen, Y., Ni, Q., Chen, Y., Hu, S., . . . Huang, H. (2020). Management of corona virus disease-19 (COVID-19): the Zhejiang experience. Journal of Zhejiang University (medical science), 49 (1), $0-0$.

Xu, X.-W., Wu, X.-X., Jiang, X.-G., Xu, K.-J., Ying, L.-J., Ma, C.-L., . . . Gao, H.-N. (2020). Clinical findings in a group of patients infected with the 2019 novel coronavirus (SARS-Cov-2) outside of Wuhan, China: retrospective case series. $b m j, 368$.

Xu, X., Chen, P., Wang, J., Feng, J., Zhou, H., Li, X., . . . Hao, P. (2020). Evolution of the novel coronavirus from the ongoing Wuhan outbreak and modeling of its spike protein for risk of human transmission. Science China Life Sciences, 63 (3), 457-460.

\section{Hosted file}


Figure 1.docx available at https://authorea.com/users/321397/articles/450673-probiotics-inviral-infections-with-a-focus-on-covid-19-a-systematic-review

\section{Hosted file}

Figure 2.docx available at https://authorea.com/users/321397/articles/450673-probiotics-inviral-infections-with-a-focus-on-covid-19-a-systematic-review

\section{Hosted file}

Figure 3.docx available at https://authorea.com/users/321397/articles/450673-probiotics-inviral-infections-with-a-focus-on-covid-19-a-systematic-review 